\title{
Preparation of siRNA-PLGA/Fab囚-PLGA mixed micellar system with target cell-specific recognition
}

Mai Hazekawa ( $\nabla$ mhaze@fukuoka-u.ac.jp )

Fukuoka University

Takuya Nishinakagawa

Fukuoka University

\section{Takeshi Mori}

Mukogawa Women's University

Miyako Yoshida

Mukogawa Women's University

Takahiro Uchida

Mukogawa Women's University

Daisuke Ishibashi

Fukuoka University

\section{Research Article}

Keywords: Fab囚, siRNA, PLGA, micelle, targeting

Posted Date: January 11th, 2021

DOl: https://doi.org/10.21203/rs.3.rs-140786/v1

License: (c) (i) This work is licensed under a Creative Commons Attribution 4.0 International License. Read Full License

Version of Record: A version of this preprint was published at Scientific Reports on August 18th, 2021. See the published version at https://doi.org/10.1038/s41598-021-96245-3. 


\section{Abstract}

Small interfering RNAs (siRNAs) are susceptible to nucleases and degrade quickly in vivo. Moreover, siRNAs demonstrate poor cellular uptake and cannot cross the cell membrane because of its polyanionic characteristics. To overcome these challenges, an intelligent gene delivery system that protects siRNAs from nucleases and facilitates siRNA cellular uptake is required. We previously reported the potential of siRNA-poly(D,L-lactic-co-glycolic acid; PLGA) micelles as an effective siRNA delivery tool in a murine peritoneal dissemination model by local injection. However, there was no effective formulation for siRNA delivery to target cells via intravenous injection. This study aimed to prepare siRNA-PLGA/FabD-PLGA mixed micelles for siRNA delivery to target floating cells and evaluate its formulation in vitro. As the target siRNA protein in CEMx174, CyclinB1 levels were significantly reduced when siRNA-PLGA/Fab囚PLGA mixed micelles were added to cells compared with siRNA-PLGA micelles. siRNA-PLGA/Fab囚PLGA mixed micelles have high cell permeability and high target cell accumulation by endocytosis because flow cytometry detected labeling micelles in target cells. This study supports siRNAPLGA/Fab囚-PLGA mixed micelles as an effective siRNA delivery tool. This formulation can be administered systemically in dosage form against target cells, including cancer metastasis or blood cancer.

\section{Introduction}

A small interfering RNA (siRNA) can recognize and degrade its complementary target mRNA in a sequence-specific manner at the post-transcriptional level ${ }^{1,2}$. Use of RNA interference (RNAi) ${ }^{3}$ to suppress target gene expression has widespread therapeutic potential. siRNA can target genes that are specific for tumor cells, thus leaving healthy, non-tumor tissue unaffected. However, developing safe and efficient carriers for siRNA delivery remains a significant challenge in animal experiments and clinical trials.

In our previous study, we developed a drug-delivery system using poly(D,L-lactic-co-glycolic acid (PLGA), which maintained the stability of the encapsulated drug and enabled the regulation of drug release $\mathrm{e}^{4,5}$. Additionally, our recent research demonstrated that polyethylenimine (LPEI)-coated siRNA-PLGA micelles were useful and safe as a siRNA delivery system by intraperitoneal injection in a mouse peritoneal dissemination model of ovarian cancer ${ }^{6}$; however, its specific accumulation for targeting cells by intravenous injection remains unclear.

Generally, small nanoparticles (20-100 nm), including micelles, actively accumulate in tumor tissues through the enhanced permeability and retention (EPR) effect, which is characterized by leaky vasculature and impaired lymphatic drainage surrounding a tumor ${ }^{7,8}$. However, expecting sufficient accumulation in cancer tissue by simply using nanoparticles is unreasonable, and there is no universal formulation design for targeting carriers because the EPR effect substantially varies depending on the patient and cancer type ${ }^{9-11}$. Attaching targeting ligands to nanoparticles or modifying the surface of nanoparticles provides further advantages for drug delivery, such as high-performance targeting, increased cellular uptake, and improved therapeutic efficacy ${ }^{12-15}$. 
Numerous targeting molecules are available for attachment to nanoparticles, including small molecules, peptides, monoclonal antibodies, engineered proteins, and nucleic acid aptamers. Choosing the correct targeting ligand and conjugation chemistry is essential for a universal formulation design and may impact the therapeutic outcome ${ }^{16,17}$. Antibody-based cell-targeting molecules, such as mAb, $F(a b \rrbracket) 2$,

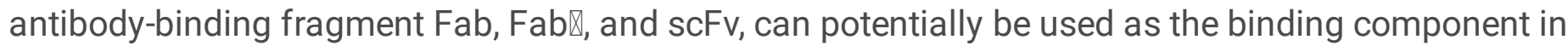
targeted nanoparticles because antibodies can be generated against a wide variety of targets with controlled affinity and specificity. Furthermore, these molecules can be produced in high yields using a cell-based expression system. There is also an advantage to reducing the particle size; because antibody fragments are smaller compared with the original size of antibodies, delivery through membranes by smaller particles is more effective. Using antibody fragments can also reduce non-specific binding by eliminating Fc interactions because many cells have receptors that can bind to the Fc region.

In this study, we followed our previous methods to prepare mixed micelles comprising the siRNA-PLGA and Fab『-PLGA hybrids, as shown in Fig. 1. The CEMx174 cell line was used as a model human floating cell line because the purpose of this study was to develop an antibody-based drug carrier that demonstrates accumulation in systemic circulation. Thus, the formulation was evaluated in vitro for targeting and intracellular uptake efficiency. This is the first paper that proposes this novel mixed-micelles type of formulation using antibody fragments for drug targeting in siRNA delivery.

\section{Results}

\section{Confirmation of Fab囚 binding activity against its antigen using flow cytometry}

$\mathrm{F}(\mathrm{abZ}) 2$ was detected by western blotting after sodium dodecyl sulphate-polyacrylamide gel electrophoresis (SDS-PAGE) was performed using a 150-kDa band and was purified using a commercially available $\mathrm{F}(\mathrm{ab} \bigotimes) 2$ preparation kit, as shown in Supplemental Figures $1 \mathrm{~A}$ and $1 \mathrm{~B}$. A functional binding analysis was performed using flow cytometry, as shown in Supplemental Figure 1C. The Fab $₫$ that was used as a component of the PLGA hybrid retained binding activity against human CD71 expressed on the surface of CEMx174 cells and was similar to fully expressed IgG activity levels.

\section{Evaluation of siRNA-PLGAFFab-PLGA mixing ratios based on intercellular uptake efficiency}

The siRNA-PLGA and Fab囚-PLGA mixing ratios were evaluated using flow cytometry based on the siRNA intracellular uptake efficiency. The fluorescence intensity of cells that were treated with various Fab囚PLGA ratios to prepare a sample that was entirely (Alexa488-siRNA)-PLGA was measured after $24 \mathrm{~h}$ of incubation. Microscopic photographs were taken before the fluorescence was measured using flow cytometry. The results of flow cytometry are shown in Figure 2.

Figure 2A shows that the peak for siRNA-PLGA/Fab囚-PLGA mixed micelles shifted more to the right compared with the peak for Fab囚-PLGA micelles. However, there were no significant differences in intracellular siRNA uptake efficiency between the various mixing ratios of the Fab囚-PLGA and siRNAPLGA; this was attributed to the reactivity of Fab囚 to CD71 highly expressed on the CEMx174 cell surface 
protein. Based on these results and those of a previous study using a ratio of $25 \%$ antibody fragment to micelles $\mathrm{mol}^{18}{ }^{18}$, the Fab囚-PLGA-to-siRNA-PLGA mixing ratio of $25 \%$ was used in this study as the minimum concentration for sufficient functionality. In the case of siRNA/Fab》-PLGA mixed micelles using the control IgG (non-specific activity to CD71), as shown in Figure 2B, the peak was overlapped by the peak of siRNA-PLGA (10:0). These results prove that the use of CD71-specific Fab区 used in this preparation improves the specific intracellular uptake of target cells.

\section{Size and surface charge of siRNA-PLGA micelles and siRNA-PLGA/Fab区-PLGA mixed micelles}

The hydrodynamic sizes and surface charges of siRNA-PLGA micelles and siRNA-PLGA/Fab囚-PLGA mixed micelles were analyzed using a dynamic light-scattering technique. As shown in Table 1, the mean diameter and zeta potential of siRNA-PLGA micelles were $113.3 \pm 0.09 \mathrm{~nm}$ and $-22.1 \mathrm{mV}$, respectively, and the mean diameter and zeta potential of siRNA-PLGA/Fab囚-PLGA mixed micelles were $112.8 \pm 0.30$ $\mathrm{nm}$ and $-42.5 \mathrm{mV}$, respectively. Fab囚-PLGA micelles were also prepared as a control. The mean diameter and zeta potential of Fab囚-PLGA micelles were $170.9 \pm 0.26 \mathrm{~nm}$ and $-68.5 \mathrm{mV}$, respectively.

\section{Cyclin B1 expression levels in CEMx174 cells treated with siRNA-PLGA micelles and siRNA-PLGAFab区- PLGA mixed micelles}

siRNA sequences that exhibited potent knockdown effects in a dose-dependent manner when delivered with transfection reagents were selected for subsequent experiments using micellar delivery without transfection agents. The cyclin B1 levels in CEMx174 cells treated with siRNA-PLGA micelles and siRNA-PLGA/FabD-PLGA mixed micelles were then evaluated with the help of western blotting. As shown in Figure 3A, siRNA-PLGA micelles significantly suppressed cyclin B1 expression compared with the control, as did siRNA-PLGA/Fab区-PLGA mixed micelles. Moreover, siRNA (negative control; N.C.)PLGA micelles and Fab囚-PLGA micelles, which do not include cyclin B1-targeting siRNA, did not suppress cyclin B1 expression as seen in the control group.

In the evaluation of the cyclin B1 knockdown effect using the CEMx174 cell line, mixed micelles showed a significant protein knockdown effect compared with non-mixed micelles.

\section{Cell viability assay}

Cell viability was evaluated to determine the effects of cyclin B1 downregulation on cell proliferation. As shown in Figure 3B, treatment with siRNA-PLGA micelles and siRNA-PLGA/FabD-PLGA mixed micelles, particularly the latter, significantly suppressed cell proliferation compared with the control.

\section{Cell accumulation of the siRNA-PLGA hybrid or Fab囚-PLGA hybrid using labeled PLGA in the CEMx174 cell line culture medium}

It was considered that Fab囚-PLGA could be present only on the cell surface due to antibody-antigen reaction. From the results of protein quantification using western blotting as shown in Figure 3 , we can see that siRNA was delivered efficiently to the cells via the mixed micelle system. However, the 
localization of these hybrids cannot be determined using flow cytometry alone, as shown in Figure 2. Further, to evaluate the cell accumulation of the micelles when added to the cells, fluorescence intensity was measured using fluorescently labeled micelles comprising fluorescein isothiocyanate (FITC)-labeled PLGA via a confocal laser scanning microscope. Localization was observed by a confocal microscope to evaluate the differences between mixed and non-mixed micelles conjugating in the four types of PLGA, as shown in Figures $4 A$ and $4 B$, respectively. The fluorescence intensity induced by siRNA or PLGA in the two types of micelles was detected not only on the cell surface but also inside the cells.

\section{Discussion}

In this study, we confirmed that the antibodies did not lose their activity due to fragmentation as shown in Supplemental Fig. 1C, although fragmentation might inhibit their activity depending on the method and antibody stability ${ }^{19}$. Thus, the mixed micelle formulation prepared in this study is designed as a novel dosage form that recognizes target molecules like CD71 wherein the antibody activity is maintained.

The average particle size of the mixed micelles prepared in this study was approximately $110 \mathrm{~nm}$, and the surface charge was approximately - $30 \mathrm{mV}$, but the Fab囚-PLGA micelle particle size was larger than other micelles. The particle size difference was suggested to result from the intermolecular force of the hybrid during micelle formation; this force varies greatly depending on the type of monomer and solvent ${ }^{20}$. The mixed micelle is almost the same size as a single non-mixed micelle and mixing does not interfere with the cell permeation efficiency.

From the results shown in Fig. 3 or those of our previous study ${ }^{6}$, approximately $100 \mathrm{~nm}$ particles, including siRNA-PLGA micelles, undergo endocytosis. Endocytosis is thought to be promoted by

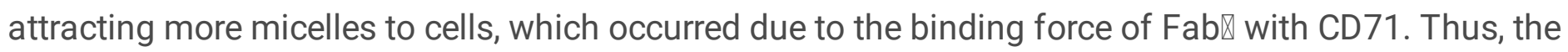
siRNA transfection efficiency of the cells increased. It was speculated that not only endocytosis but also the promoting of CD71 internalization enhances the knockdown effect as an additive action ${ }^{21,22}$. Therefore, it was suggested that the intracellular uptake of mixed micelles involves target receptor internalization, which then has an additive effect on endocytosis promotion by the mixed micelle with an antibody fragment against CD71.

Cyclin B1, a target protein of siRNA, was significantly involved in the cell cycle and regulated cell proliferation ${ }^{23}$. Cyclin B1 may also be a key target for anti-proliferative strategies, and siRNA used in cyclin B1 targeting approaches has been proposed as a useful antiproliferative therapy ${ }^{24-26}$. Therefore, these results have a positive correlation between the protein expression level and cell proliferation and are appropriate. Furthermore, it is assumed that protein knockdown by siRNA leads to the suppression of cell growth.

These results show that micelles consisting of siRNA-loaded PLGA conjugates were taken up into the cell by endocytosis, and siRNA was reliably delivered intracellularly. Furthermore, the Fab》-PLGA conjugate was also incorporated into the cell as a micelle particle rather than staying only on the cell surface. From 
these results, it was determined that the mixed micelles recognized target cells and efficiently delivered siRNA into the cells without inhibiting the intercellular uptake of micelle particles. It has been suggested that mixed micelles be used as novel drug carriers that recognize target cells or as an efficient drugdelivery device to deliver siRNA into the cells.

To our knowledge, this was the first study to show that siRNA-PLGA/Fab囚-PLGA mixed micelles can effectively deliver siRNA to cancer cells and recognize cancer cells in vitro. In addition, our strategy for targeting cancer cells using antibody-based drugs could offer new information about developing nucleic acid medications that use siRNA as a therapeutic approach without the side effects associated with anticancer drugs. This study targeted CEMx174, a lymphocyte. Therefore, the mixed micelle proposed in this study is expected to target solid tumors or floating cells like blood cancers or immune cells by customizing the antibody target. The design formulated for targeting in this study can be applied to various diseases because the amino acid sequence of $\mathrm{Fab} \mathbb{\mathrm { a }}$ and the base sequence of siRNA for targeting can be customized according to the therapeutic approach required for a particular disease. As a next step, we plan to examine the therapeutic effect in an animal model.

\section{Methods}

\section{Materials}

Poly(D,L-lactic-co-glycolic acid) (PLGA7510; $\left.\mathrm{M}_{\mathrm{W}}, 10,000\right)$ was purchased from Wako Pure Chemical Industries, Ltd. (Osaka, Japan). Human cyclin B1-specific siRNA, which was modified with a thiol group at the 3囚 end of the sense or antisense strand, was purchased from Thermo Fisher Scientific, Inc. (Waltham, MA, USA). The siRNA sequences were as follows: cyclin B1 siRNA sense: $5 \llbracket-G G C$ GAA GAU CAA CAU GGC ATT-3囚; and cyclin B1 siRNA antisense: 5囚-UGC CAU GUU GAU CUU CGC CTT-3囚 (17). Negative control siRNA was also purchased from Thermo Fisher Scientific, Inc. (Waltham). Fetal calf serum (FCS) and Roswell Park Memorial Institute (RPMI) 1640 were purchased from Gibco (Cergy-Pontoise, France). Antihuman CD71 monoclonal antibody, phycoerythrin (PE) anti-human CD71 antibody (Clone: OKT9) and were purchased from Thermo Fisher Scientific Inc. Normal mice IgG1 kappa monoclonal, which was used as the control IgG, was purchased from Abcam (ab91353, Cambridge, UK). A Pierce IgG F(ab凹)2 preparation kit was purchased from Thermo Fisher Scientific Inc. Cell Counting Kit-8 (CCK-8) reagent was purchased from Dojindo Laboratories (Tokyo, Japan). Alexa488-labeled siRNA was purchased from Thermo Fisher Scientific, Inc. (Waltham). Cy5-labeled siRNA was purchased from Nippon Gene (Toyama, Japan). FITC-labeled PLGA was synthesized by the NARD Institute, Ltd. (Amagasaki, Japan).

\section{Preparation of siRNA-PLGA micelles}

The siRNA-PLGA hybrid block copolymer was synthesized as previously described ${ }^{6}$. Antisense and sense $3 \otimes$ thiol-modified siRNAs were conjugated to PLGA-3-(2-pyridyldithio) propionyl hydrazide (PDPH) via disulfide exchange reaction. The synthesized siRNA-PLGA hybrids were expected to form self- 
assembled micelles in aqueous solutions, thereby resulting in a substantially increased charge density of clustered siRNAs on the outer shell (Figs. 1A, 1C).

\section{Preparation and isolation of the $F(a b \varangle) 2$ fragment from the whole antibody}

$A F(a b) 2$ preparation kit was used to cleave $F(a b \rrbracket) 2$ from the whole antibody in accordance with the manufacturer's protocol. In this study, anti-human CD71 antibody was used as a model for targeting the Fab囚 fragment because CD71 is expressed highly in the CEMx174 cell line. The yield solution was prepared in SDS-PAGE loading dyes and analyzed using SDS-PAGE.

\section{Preparation of Fab区-PLGA micelles and siRNA-PLGAFab区-PLGA mixed micelles}

$\mathrm{F}(\mathrm{abZ}) 2$ fragments of the antibody in phosphate-buffered saline (PBS; $0.5 \mathrm{mg} / \mathrm{mL}, 400 \mu \mathrm{L}$ ) were mixed with dithiothreitol (DTT) $(0.5 \mathrm{mM}, 240 \mu \mathrm{L})$ and incubated at $37^{\circ} \mathrm{C}$ for $30 \mathrm{~min}^{12}$ to reduce $\mathrm{F}(\mathrm{ab} \otimes) 2$

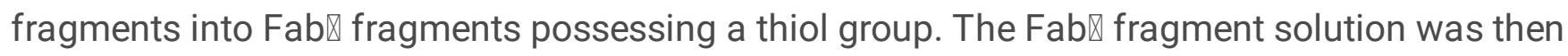
conjugated to PLGA-PDPH via disulfide exchange reaction in the same manner that was used to prepare the siRNA-PLGA hybrid. The siRNA-PLGA and Fab囚-PLGA hybrid solutions were mixed at a volume ratio of $4: 1$ in accordance with previous methods ${ }^{19}$ (Figs. 1B, 1D).

\section{Morphology and surface charge of the siRNA-PLGA micelles and siRNA-PLGA/FabD-PLGA mixed micelles}

The sizes and surface charges of the siRNA-PLGA micelles and siRNA-PLGA/Fab区-PLGA mixed micelles were measured using a dynamic light-scattering instrument (Zetasizer; Malvern Instruments, Ltd., Malvern, UK). Both these types of micelles (300 pmol) were dissolved in $500 \mu \mathrm{L}$ distilled water. The effective hydrodynamic diameters and zeta potentials of these micelles were measured in triplicate.

\section{Cell culture}

The human T-B hybrid cell line CEMx174 was a gift from the American Type Culture Collection (ATCC; catalog CRL-1991, Rockville, Maryland, USA). CEMx174 cells were cultured in an RPMI 1640 medium (Gibco) supplemented with $10 \% \mathrm{FCS}$ at $37^{\circ} \mathrm{C}$ in an atmosphere of $95 \%$ air and $5 \% \mathrm{CO}_{2}$.

\section{Cyclin B1 gene silencing by siRNA-PLGA micelles or siRNA-PLGAFFab-PLGA mixed micelles without a transfection reagent}

CEMx174 cells were seeded in a 24-well plate at a density of $5 \times 10^{5}$ cells per well to measure the relative cyclin B1 expression by western blotting. The siRNA-PLGA micelles or siRNA-PLGA/Fab囚-PLGA mixed micelles ( $80 \mathrm{pmol}$ each based on the siRNA content) were added to the well. After $48 \mathrm{~h}$ of incubation, the cells were lysed with $1 \%(\mathrm{w} / \mathrm{v})$ Triton X-100 solution in PBS and centrifuged to remove cell debris.

\section{Western blot analysis}


To detect the cyclin B1 protein, cells were lysed in a lysis buffer. The total protein concentrations of the lysates were measured using bicinchoninic acid (BCA) assay (Pierce Biotechnology, Rockford, IL, USA). Cell lysates containing equal amounts of protein were separated by SDS-PAGE (Bio-Rad, Hercules, CA, USA), and protein bands were transferred to polyvinylidene difluoride membranes (Bio-Rad). The membranes were blocked with Blocking One (Nacalai Tesque, Inc., Kyoto, Japan) overnight at $4^{\circ} \mathrm{C}$ and then incubated for $1 \mathrm{~h}$ at $22^{\circ}-25^{\circ} \mathrm{C}$ with anti-cyclin B1 antibody (ab2949; Abcam, Cambridge, UK) or an anti-glyceraldehyde 3-phosphate dehydrogenase antibody (ACR001PT; Acris Antibodies, Inc., San Diego, CA, USA) at a 1:1000 and 1:10,000 dilution, respectively, in a blocking solution. After washing three times, the membranes were incubated for $1 \mathrm{~h}$ at $22^{\circ} \mathrm{C}-25^{\circ} \mathrm{C}$ with a secondary antibody (horseradish peroxidaseconjugated species-specific antibody). Immunoreactive bands were visualized with ImmunoStar LD (Wako Pure Chemical Industries).

\section{Cell viability assay}

CyclinB1 gene knockdown effect on the proliferation of CEMx174 cells was evaluated using the CCK-8 reagent. Briefly, $1 \times 10^{4}$ cells in $100 \mu \mathrm{L}$ RPMI 1640 containing 10\% FCS were seeded into 96-well plates. Each well was then treated with $50 \mu \mathrm{L}$ of micelles for $48 \mathrm{~h}$ or with $50 \mu \mathrm{L}$ PBS as a control. Then, $15 \mu \mathrm{L}$ CCK-8 reagent was added to each well, and the cells were further incubated at $37^{\circ} \mathrm{C}$ for $2 \mathrm{~h}$. Absorbance was measured using a microplate reader at test and reference wavelengths of 450 and $655 \mathrm{~nm}$, respectively, to evaluate the relative viability of the treated cells.

\section{Flow cytometry}

\section{Evaluation of the binding activity of the antibody fragment}

IgG, $F(a b \rrbracket) 2$, or Fab》 was added to CEMx 174 cells blocked by $40 \%$ normal goat serum at a density of $5 \times$ $10^{5}$ cells per tube. After a 30-min incubation, cells were washed using a washing buffer, and PE-labeled secondary antibody against IgG was added to the cells. After a 30-min incubation, the fluorescence intensity of the cells was determined using a flow cytometer (NovoCyte, ACEA Biosciences, Inc., San Diego, CA, USA).

\section{Evaluation of the mixing volume ratio in siRNA-PLGAFab囚-PLGA mixed micelles}

CEMx174 cells were seeded in a 24-well plate at a density of $5 \times 10^{5}$ cells per well. siRNA-PLGA mixed with Fab囚-PLGA was added to the well at a volume ratio of 0:0, 10:0, 10:2, 10:5, 10:8, or 0:10. The 10volume ratio of siRNA-PLGA was determined to be 80 pmol based on the siRNA content, whereas the 8volume ratio of $\mathrm{Fab} \otimes-P L G A$ was $5 \mu \mathrm{g}$ based on the Fab囚 content. Cells were used to evaluate the fluorescence intensity after $24 \mathrm{~h}$ of incubation. The fluorescence intensity of Alexa488-siRNA was detected using a flow cytometer.

\section{Localization of the siRNA-PLGA or FabD-PLGA conjugate consisting of mixed micelles after cellular uptake into the CEMx174 cells}


CEMx 174 cells were seeded in a 24-well plate at a density of $5 \times 10^{5}$ cells per well. The four types of siRNA-PLGA/Fab囚-PLGA mixed micelles were added to the well to evaluate whether each part of the micelle was taken up into the cells. After $24 \mathrm{~h}$ of incubation, the cells were used to evaluate the fluorescence intensity. In this evaluation, Cy5-labeled siRNA or FITC-labeled PLGA was used to prepare three types of a siRNA-PLGA hybrids, (Cy5-labeled siRNA)-PLGA, siRNA-(FITC-labeled PLGA), and Fab区(FITC-labeled PLGA). The fluorescence intensity of Cy5 and FITC was observed using a confocal microscope (LMS710, Carl Zeiss, Oberkochen, Germany) with a 40x objective lens to evaluate the localization of the fluorescence intensity in the cells by slicing to distinguish between the surface and cell interior. Image analysis was performed using LSM Software ZEN 2009 (Carl Zeiss,).

\section{Statistical analysis}

Values were expressed as mean \pm standard deviation (SD) $(n=3-5)$. To determine the differences among the groups, data were evaluated for statistical significance using the Bonferroni test. Overall significance was determined using a one-way repeated measures analysis of variance (ANOVA). A $p$ value of $<0.05$ was considered to be statistically significant.

\section{Data availability}

The datasets generated and/or analyzed during the current study are available from the corresponding author on reasonable request.

\section{Declarations}

\section{Acknowledgments}

We thank Tomoyo Kawakubo-Yasukochi, and Manabu Nakashima for valuable discussions. This work was supported by JSPS KAKENHI Grant Number JP20K07214; Fukuoka Foundation for Sound Health Cancer Research Fund; and funds (No. 181045) from the Central Research Institute of Fukuoka University. We also thank Edanz Group (https://en-author-services.edanz.com/ac) for editing a draft of this manuscript.

\section{Author contributions}

M.H. conceived and designed the project. M.H. and T.N. performed all the experiments involving preparation of micelles, data analysis, and figure preparations. T.M. measured the particle size and zeta potential using Zetasizer. M.H. and T.N. wrote the manuscript. M.Y., T.U., and D.I. reviewed and edited the manuscript. All authors read and approved the manuscript.

\section{Competing interests}

The authors declare no competing interests. 


\section{References}

1. Fire, A., et al. Potent and specific genetic interference by double-stranded RNA in Caenorhabditis elegans. Nature. 391(6669), 806-811 (1998).

2. Elbashir, S.M., et al Duplexes of 21-nucleotide RNAs mediate RNA interference in cultured mammalian cells. Nature. 411(6836), 494-498 (2001).

3. Tuschl, T., Zamore, P.D., Lehmann, R., Bartel, D.P. \& Sharp, P.A., Targeted mRNA degradation by double-stranded RNA in vitro. Gene Dev. 13(24), 3191-3197 (1999).

4. Hazekawa, M., Morihata, K., Yoshida, M., Sakai, Y. \& Uchida, T., The angiogenic effect of ONO-1301, a novel long-acting prostacyclin agonist loaded in PLGA microspheres prepared using different molecular weights of PLGA, in a murine sponge model. Drug Dev Ind Pharm. 40(11), 1435-1442 (2014).

5. Hazekawa, M., Kojima, H., Haraguchi, T., Yoshida, M. \& Uchida, T., Effect of self-healing encapsulation on the initial burst release from PLGA microspheres containing a long-acting prostacyclin agonist, ONO-1301. Chem Pharm Bull. 65(7), 653-659 (2017).

6. Hazekawa, M., Nishinakagawa, T., Kawakubo-Yasukochi, T. \& Nakashima, M., Glypican-3 gene silencing for ovarian cancer using siRNA-PLGA hybrid micelles in a murine peritoneal dissemination model. J Pharmacol Sci.139 (3): 231-239 (2019).

7. Cabal, H., et al. Accumulation of sub-100 nm polymetric micelles in poorly permeable tumors depends on size. Nat. Nanotechnol, 6812, 815-823 (2011)

8. Maeda, H., The enhanced permeability and retention (EPR) effect in tumor vasculature : the key role of tumor-selective macromolecular drug targeting. Adv. Enzyme Regul, 41, 189-207 (2001).

9. Bae, Y.H., Drug targeting and tumor heterogeneity, J. Controlled Release, 133(1), 2-3 (2009).

10. Nakamura, H., Jun, F. \& Maeda, H., Development of next-generation macromolecular drugs based on the EPR effect : challemges and pitfalls. Expert Opin. Drug Delivery, 12(1), 53-64 (2015)

11. Theek, B., Gremse, F., kunjachan, S., Fokong, S. \& Pola, R., Pechar Characterizing EPR-mediated passive drug targeting using constrast-enhanced functional ultrasound imaging. J. Controlled Release, 182, 83-89 (2014)

12. Ahn, J., et al. Antibody fragment-conjugated polymetric micelles incorporating platinaum drugs for targeted therapy of pancreatic cancer. Biomaterials,39, 23-30 (2015).

13. Palanca-Wessels, M.C., et al., Anti-CD22 antibody targeting of pH-responsive micelles enhances small interfering RNA delivery and gene silencing in lymphoma cells. Mol. Ther., 19(8), 1529-1537 (2011).

14. Chan, D.P., Owen, S.C. \& Shoichet, M.S., Double click: dual functionalized polymetric micelles with antibodies and peptides. Bioconjugate Chem, 24(1), 105-113 (2013).

15. Miura, Y. et al, Cyclic RGD-linked polymetric micelles for targeted delivery of platinum anticancer drugs to glioblastoma through the blood-brain tumor barrier. ACS Nano, 7(10), 8583-8592 (2013). 
16. Steichen, S.D., Caldorera-Moore, M. \& Peppas, N.A., A review of current nanoparticle and targeting moieties for the delivery of cancer therapeutics. Eur. J. Pharm. Sci,48(3), 416-427 (2013).

17. Friedman, A.D., Claypool, S.E. \& Liu, R., The smart targeting of nanoparticles. Curr Pharm Des, 19(35), 6315-6329 (2013).

18. Yamamoto, Y., et al., Enhanced antitumor effect of anti-tissue factor antibody-conjugated epirubicinincorporating micelees in xenograft models, Cancer Sci, 106(5), 627-634 (2015).

19. Behring, S., Hänsch, R., Helmsing, S., Schirrmann, T. \& Schubert, M., Screening for scFv-fragments that are stable and active in the cytosol, Hum Antibodies, 2020, DOI:10.3233/HAB-200402

20. Yu T, Lin M, Wan J, Cao X, Molecular interaction mechanisms in reverse micellar extraction of microbial transglutaminase, $J$ chromatogr $A, 1511,25-36$ (2017).

21. Jabara, H.H., et al, A missense mutation in TFRC, encoding transferrin receptor 1 , causes combined immunodeficiency. Nat Genet, 48 (1), 74-78 (2016).

22. Roldán, J.S., Martínez, M.G., Forlenza, M.B., Whittaker, G.R. \& Candurra, N.A. Human transferrin receptor triggers an alternative Tacaribe virus internalization pathway. Arch Virol. 161(2), 353-363 (2016).

23. Crombez, L., et al., Targeting cyclinB1 through peptide-based delivery of siRNA prevents tumor growth, Nucleic Acids Res, 37(14), 4559-4569 (2009).

24. Goga, A., Yang, D., Tward, A.D., Morgan, D.O. \& Bishop, J.M., Inhibition of CDK1 as a potential therapy for tumors over-expressing MYC. Nat Med, 26(55), 7611-7619 (2007).

25. Yuan, J., et al., Stable gene silencing of cyclin B1 in tumor cells increases susceptibility to taxol and leads to growth arrest in vivo, Oncogene, 25(12), 1753-1762 (2006).

26. Morris, M.C., et al., A non-covalent peptide-based carrier for in vivo delivery of DNA mimics, Nucleic Acids Res, 35(7), e49 (2007).

\section{Tables}

Table 1. Particle sizes and zeta potential values of siRNA-PLGA micelles, siRNA-PLGA/Fab》-PLGA mixed micelles, and Fab囚-PLGA micelles. Data are presented as the mean $\pm S D(n=3)$.

\begin{tabular}{|l|c|c|}
\hline \multicolumn{1}{|c|}{ sample } & Particle size $(\mathbf{n m})$ & Zeta potential $(\mathrm{mV})$ \\
\hline siRNA-PLGA & $113.3 \pm 0.09$ & -22.1 \\
\hline siRNA-PLGA/Fab'-PLGA & $112.8 \pm 0.30$ & -42.5 \\
\hline Fab'-PLGA & $170.9 \pm 0.26$ & -68.5 \\
\hline
\end{tabular}

\section{Figures}


<smiles>CC(C)OC(=O)C(C)OC(C)C(=O)NNC(=O)CCSSC1CCCC1</smiles>

(C)

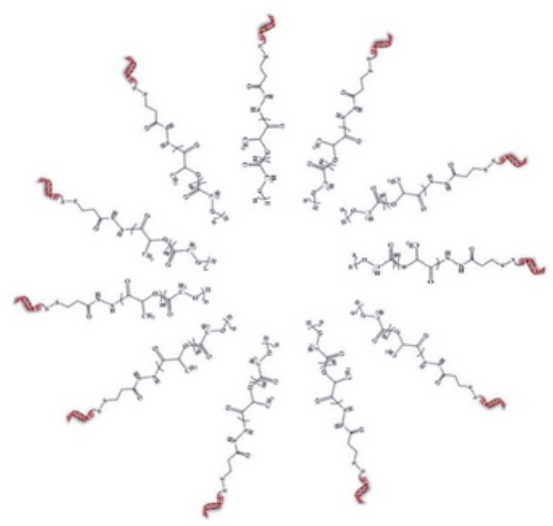<smiles>C#C[As]SSCCC(=O)NNC(=O)C(C)OC(=O)C(C)OCC(C)(C)C</smiles>

(D)

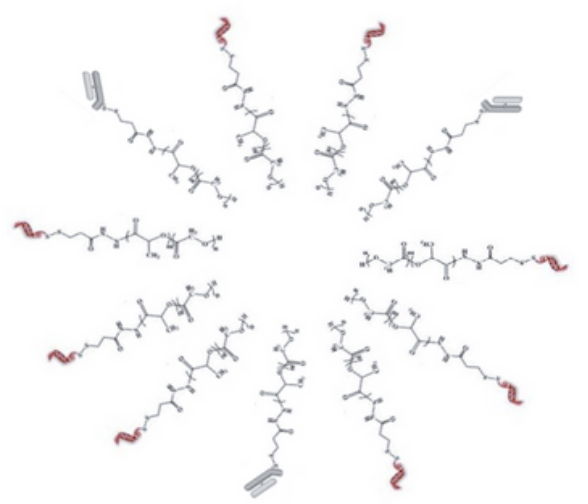

\section{Figure 1}

Structure of the siRNA-PLGA (A) and Fab囚-PLGA hybrids (B). The siRNA-PLGA hybrid (A) selfassociates above the critical micelle concentration to form (C) in an aqueous solution. (D) was formed by self-assembly when (A) was mixed with (B) in an aqueous solution. 
(A) siRNA-PLGA : Fab'(CD71)-PLGA

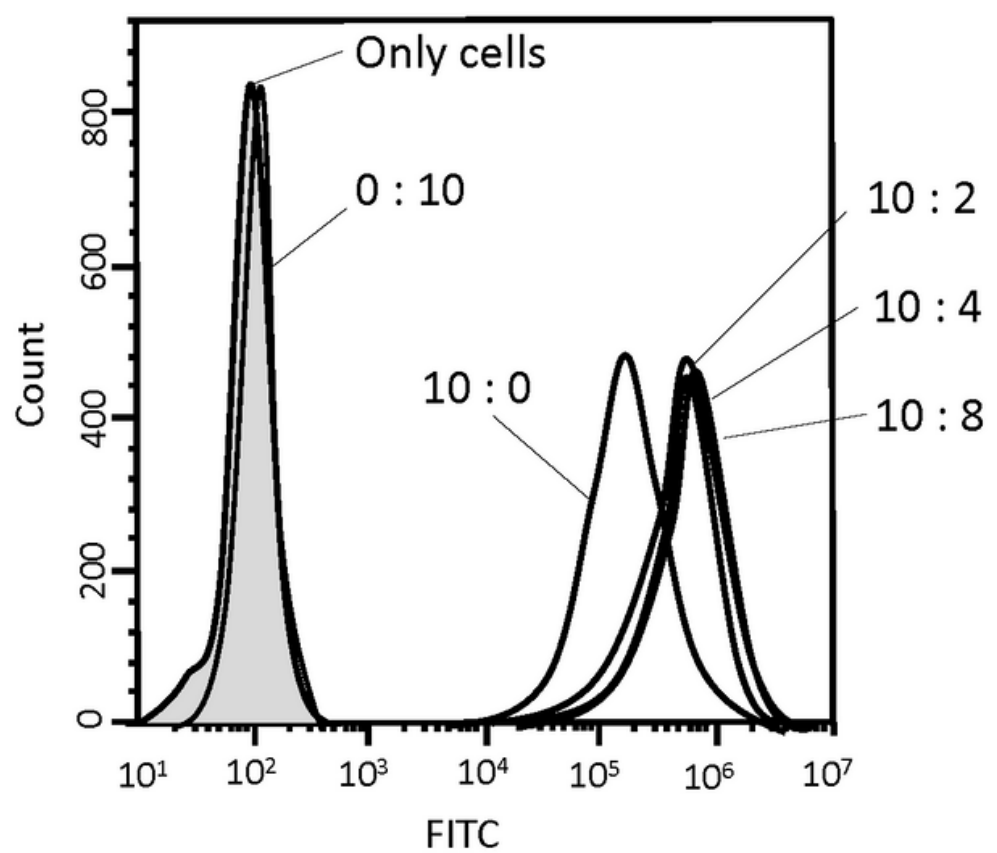

(B) siRNA-PLGA : Fab'(N.C.)-PLGA

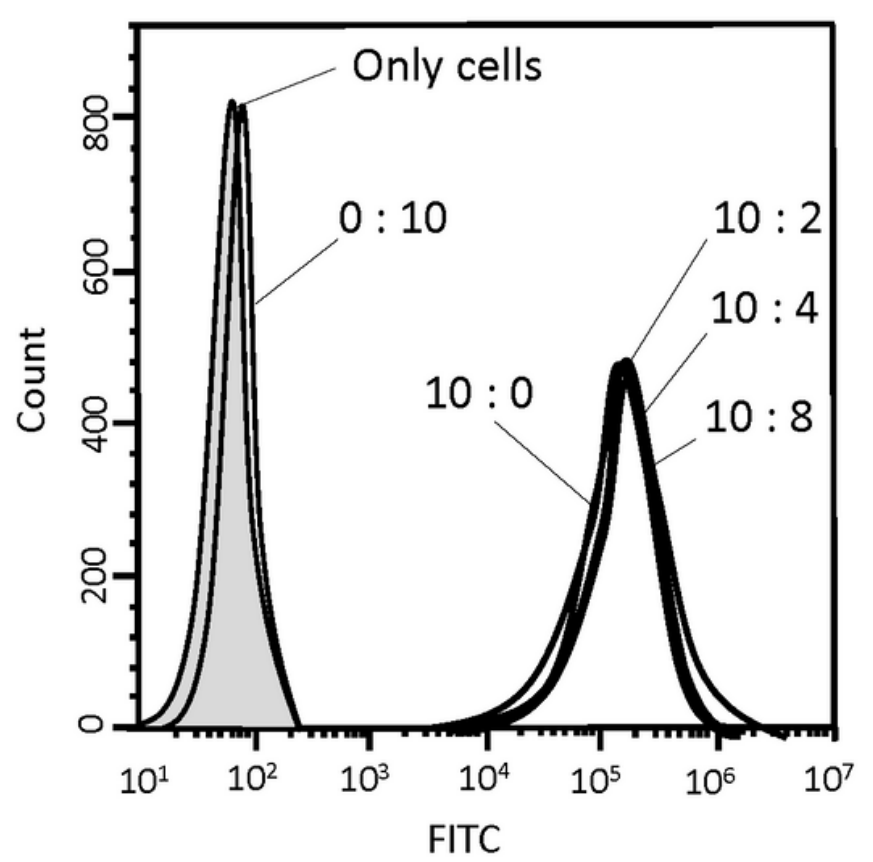

Figure 2

Flow cytometric measurement of the fluorescence intensity of CEMx 174 cells that were transfected Alexa488-siRNA using various mixing weight ratios of siRNA-PLGA and Fab区-PLGA mixed micelles. The mixing volume ratio at the side of peak in the figure is shown as (Alexa488-siRNA)-PLGA:Fab『(anti CD71 Ab)-PLGA (A) and (Alexa488-siRNA)-PLGA:Fab囚(normal mouse lgG as a negative control)-PLGA (B), respectively. 
(A)
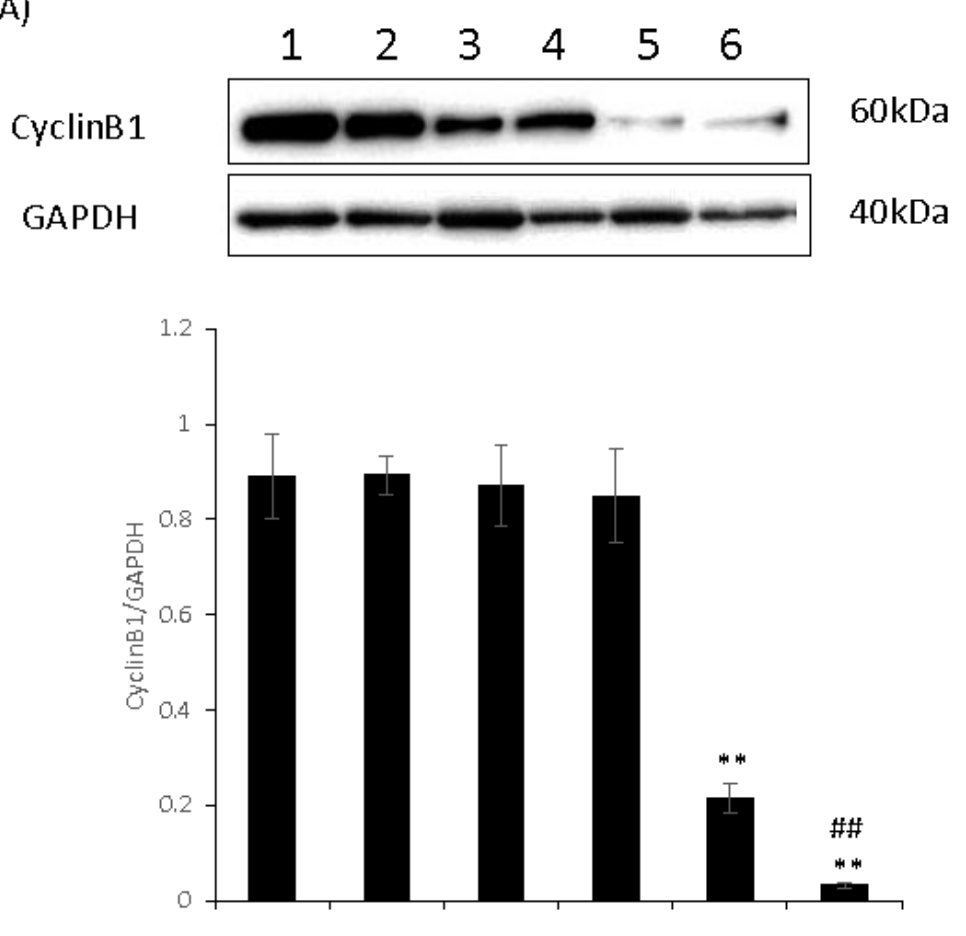

(1)
(3)
(5)

(6)
(B)

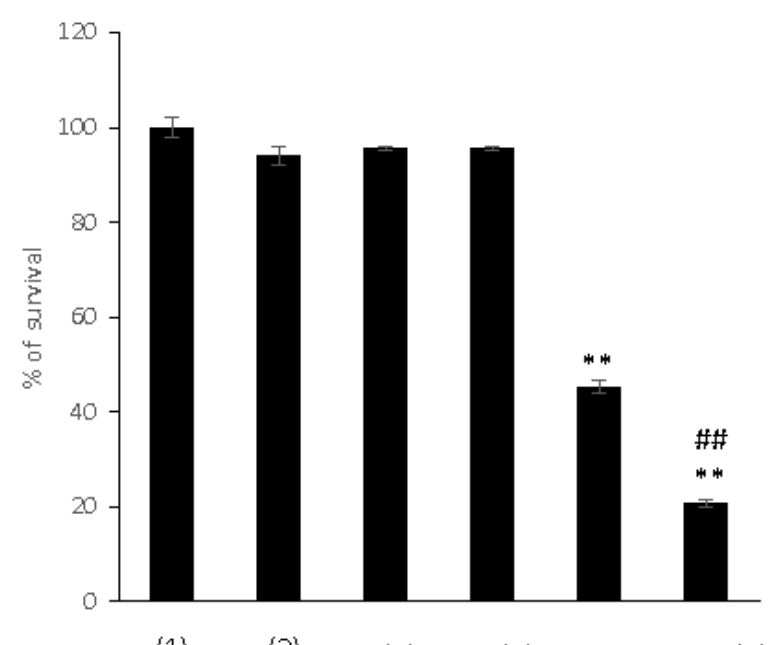

(1)

(3)

(4)

(5)

(6)

\section{Figure 3}

(A) Cyclin B1 levels in CEMx174 cells treated with siRNA-PLGA micelles and siRNA-PLGA/Fab》-PLGA mixed micelles in western blotting analysis. (1) control, (2) siRNA (N.C.), (3) siRNA, (4) siRNA (N.C.)PLGA, (5) siRNA-PLGA, (6) siRNA-PLGA/Fab凹-PLGA. Data are presented as the mean $\pm S D(n=5) . * \star p<$ 0.01 versus control. \#\#p < 0.01 versus siRNA-PLGA (Bonferroni test/ANOVA) (B) Cell viability of CEMx174 cells treated with siRNA-PLGA micelles and siRNA-PLGA/Fab囚-PLGA mixed micelles. (1) control, (2) siRNA (N.C.), (3) siRNA, (4) siRNA (N.C.)-PLGA, (5) siRNA-PLGA, (6) siRNA-PLGA/Fab区PLGA. Data are presented as the mean $\pm S D(n=3) .{ }^{* *} p<0.01$ versus control. \#\#p $<0.01$ versus siRNAPLGA group (Bonferroni test/ANOVA) 
(A)

FITC
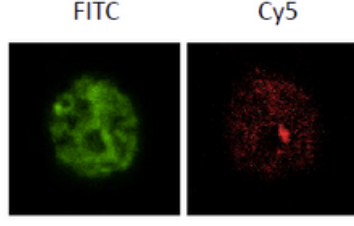

FITC/Cy5

SIRNA(CY5)-PLGA(FITC)

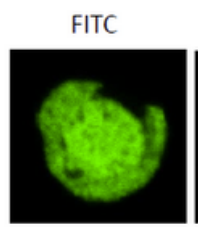

Cy5

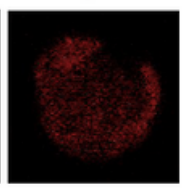

FITC/Cy5

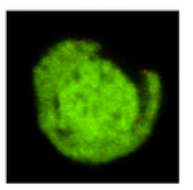

(B) SiRNA-PLGA(FITC)
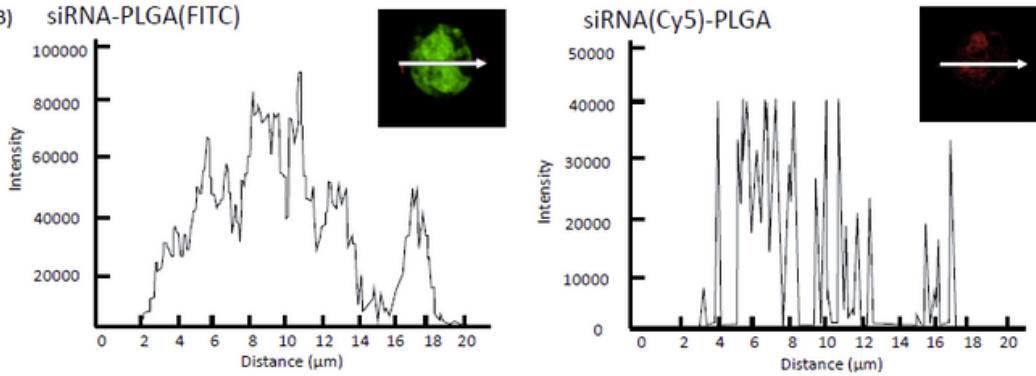

Figure 4

(A) Intracellular uptake of siRNA-PLGA, as shown in the upper line labeled with Cy5 or FITC, and siRNAPLGA/Fab囚-PLGA mixed micelles consisting of (Cy5-labeled siRNA)-PLGA and Fab》-(FITC-labeled PLGA), as shown in lower line in CEMx174 cells using a confocal laser microscope. (B) Image analysis of the fluorescence intensity of CEMx174 cells in the cross section with a white arrow using three types of labeling conjugates to evaluate the localization of these conjugates.

\section{Supplementary Files}

This is a list of supplementary files associated with this preprint. Click to download.

- Supplhazekawa210106.pdf 